\title{
On the size of Kakeya sets in finite vector spaces
}

\author{
Gohar Kyureghyan * \\ Institute of Algebra and Geometry \\ Otto-von-Guericke University Magdeburg \\ 39106 Magdeburg, Germany \\ gohar.kyureghyan@ovgu.de \\ Peter Müller \\ Institute for Mathematics \\ University of Würzburg \\ Campus Hubland Nord, 97074 Würzburg, Germany \\ peter.mueller@mathematik. uni-wuerzburg. de \\ Qi Wang \\ Institute of Algebra and Geometry \\ Otto-von-Guericke University Magdeburg \\ 39106 Magdeburg, Germany \\ qi . wang@ovgu.de
}

Submitted: Mar 7, 2013; Accepted: Aug 29, 2013; Published: Sep 6, 2013

Mathematics Subject Classifications: 11T30, $11 \mathrm{~T} 06$

\begin{abstract}
For a finite field $\mathbb{F}_{q}$, a Kakeya set $K$ is a subset of $\mathbb{F}_{q}^{n}$ that contains a line in every direction. This paper derives new upper bounds on the minimum size of Kakeya sets when $q$ is even.
\end{abstract}

Keywords: Kakeya set; finite vector space; Gold power function

\section{Introduction}

Let $\mathbb{F}_{q}$ be a finite field with $q$ elements. A Kakeya set $K \subset \mathbb{F}_{q}^{n}$ is a set containing a line in every direction. More formally, $K \subset \mathbb{F}_{q}^{n}$ is a Kakeya set if and only if for every $\mathbf{x} \in \mathbb{F}_{q}^{n}$,

*The first and third authors thank Vsevolod Lev for bringing their attention to [7]. The third author is supported by the Alexander von Humboldt (AvH) Stiftung/Foundation. 
there exists $\mathbf{y} \in \mathbb{F}_{q}^{n}$ such that $\left\{\mathbf{y}+t \mathbf{x}: t \in \mathbb{F}_{q}\right\} \subset K$. Wolff in [11] asked whether a lower bound of the form $|K| \geqslant C_{n} \cdot q^{n}$ holds for all Kakeya sets $K$, where $C_{n}$ is a constant depending only on $n$. Dvir [2] first gave such a lower bound with $|K| \geqslant(1 / n !) q^{n}$. Later Dvir, Kopparty, Saraf and Sudan improved the lower bound to $|K| \geqslant\left(1 / 2^{n}\right) q^{n}$ in [4] (see also [10]). It was shown in [4] that for any $n \geqslant 1$ there exists a Kakeya set $K \subset \mathbb{F}_{q}^{n}$ with

$$
|K| \leqslant 2^{-(n-1)} q^{n}+O\left(q^{n-1}\right) .
$$

For more information on Kakeya sets, we refer to a recent survey [3].

When $q$ is bounded and $n$ grows, bound (1) is weak, and some recent papers improved the $O$-term in it to give better upper bounds for this case. The best currently known bound was obtained by Kopparty, Lev, Saraf and Sudan in [5], following the ideas from [10, 4] (see also $[9])$ :

Theorem 1. [5, Theorem 6] Let $n \geqslant 1$ be an integer and $q$ a prime power. There exists a Kakeya set $K \subset \mathbb{F}_{q}^{n}$ with

$$
|K|< \begin{cases}2\left(1+\frac{1}{q-1}\right)\left(\frac{q+1}{2}\right)^{n} & \text { if } q \text { is odd, } \\ \frac{3}{2}\left(1+\frac{1}{q-1}\right)\left(\frac{2 q+1}{3}\right)^{n} & \text { if } q \text { is an even power of } 2, \\ \frac{3}{2}\left(\frac{2(q+\sqrt{q}+1)}{3}\right)^{n} & \text { if } q \text { is an odd power of } 2 .\end{cases}
$$

Theorem 1 was proved by constructing a Kakeya set $K \subset \mathbb{F}_{q}^{n}$ from a suitable function $f: \mathbb{F}_{q} \rightarrow \mathbb{F}_{q}$ as follows: For a given $t \in \mathbb{F}_{q}$, set

$$
I_{f}(t):=\left\{f(x)+t x \mid x \in \mathbb{F}_{q}\right\} .
$$

Further, define

$$
K:=\left\{\left(x_{1}, \ldots, x_{j}, t, 0, \ldots, 0\right) \mid 0 \leqslant j \leqslant n-1, t \in \mathbb{F}_{q}, x_{1}, \ldots, x_{j} \in I_{f}(t)\right\} .
$$

If $f$ is a non-linear function, then $K$ is a Kakeya set [5] of size

$$
|K|=\sum_{j=0}^{n-1} \sum_{t \in \mathbb{F}_{q}}\left|I_{f}(t)\right|^{j}=\sum_{t \in \mathbb{F}_{q}} \frac{\left|I_{f}(t)\right|^{n}-1}{\left|I_{f}(t)\right|-1} .
$$

Clearly, to construct a small Kakeya set, we need to find a function $f: \mathbb{F}_{q} \rightarrow \mathbb{F}_{q}$ for which the sets $I_{f}(t)$ are small. However, for a generic function $f: \mathbb{F}_{q} \rightarrow \mathbb{F}_{q}$, it is difficult to have a good estimation for the values $\left|I_{f}(t)\right|$, where $t \in \mathbb{F}_{q}$. Theorem 1 was proved by taking

- $f(x)=x^{2}$ for $q$ odd, since then $\left|I_{f}(t)\right| \leqslant(q+1) / 2$ holds for all $t \in \mathbb{F}_{q}$;

- $f(x)=x^{3}$ for $q$ an even power of 2 , since then $\left|I_{f}(t)\right| \leqslant(2 q+1) / 3$ holds for all $t \in \mathbb{F}_{q}$; 
- $f(x)=x^{q-2}+x^{2}$ for $q$ an odd power of 2 , since then $\left|I_{f}(t)\right| \leqslant 2(q+\sqrt{q}+1) / 3$ holds for all $t \in \mathbb{F}_{q}$.

Lemma 21 in [5] shows that for any $q$ and any function $f: \mathbb{F}_{q} \rightarrow \mathbb{F}_{q}$, there is an element $t \in \mathbb{F}_{q}$ such that $\left|I_{f}(t)\right|>q / 2$. Hence for $q$ odd the function $f(x)=x^{2}$ yields the best upper bound for the minimum size of Kakeya sets produced by the construction presented above. For an even $q$, the authors of [5] ask whether and to which extent the bounds of Theorem 1 can be improved by choosing a better function $f$. In this paper, we investigate this question further and derive indeed better upper bounds on the size of Kakeya sets $K \subset \mathbb{F}_{q}^{n}$, when $q$ is even. Our main result is

$$
|K|< \begin{cases}\frac{2 q}{q+\sqrt{q}-2}\left(\frac{q+\sqrt{q}}{2}\right)^{n} & \text { if } q \text { is an even power of } 2, \\ \frac{8 q}{5 q+2 \sqrt{q}-3}\left(\frac{5 q+2 \sqrt{q}+5}{8}\right)^{n} & \text { if } q \text { is an odd power of } 2 .\end{cases}
$$

When $q$ is an even power of 2 , say $q=2^{m}$, we prove (3) by determining explicitly the values $\left|I_{f}(t)\right|$ for $f(x)=x^{2^{m / 2}+1}$ and $t \in \mathbb{F}_{q}$. In the case $q$ is an odd power of 2 , the result follows with $f(x)=x^{4}+x^{3}$. Presently, it is not clear to us, whether the bounds in (3) can be improved.

The following result by Bluher [1] is used later in the paper:

Theorem 2. [1, Theorem 5.6] Let $q=2^{m}$ and $0 \leqslant i<m$ with $d=\operatorname{gcd}(i, m)$. Let $N_{0}$ denote the number of $b \in \mathbb{F}_{q}^{*}$ such that $x^{2^{i}+1}+b x+b$ has no root in $\mathbb{F}_{q}$.

(i) If $m / d$ is even, then $N_{0}=\frac{2^{d}(q-1)}{2\left(2^{d}+1\right)}$.

(ii) If $m / d$ is odd, then $N_{0}=\frac{2^{d}(q+1)}{2\left(2^{d}+1\right)}$.

\section{On Kakeya sets constructed using Gold power func- tions}

In this section, we use the Gold power functions $f(x)=x^{2^{i}+1}$ to derive upper bounds on the minimum size of Kakeya sets $K \subset \mathbb{F}_{q}^{n}$ with $q$ even. Theorem 2 allows us to determine explicitly the size of the image set $I_{f}(t):=\left\{f(x)+t x: x \in \mathbb{F}_{q}\right\}$ with $f(x)=x^{2^{i}+1}$ and $t \in \mathbb{F}_{q}$.

Proposition 3. Let $q=2^{m}, f(x)=x^{2^{i}+1} \in \mathbb{F}_{q}[x]$ with $0 \leqslant i<m$, and $d=\operatorname{gcd}(i, m)$. Set $I_{f}(t):=\left\{f(x)+t x: x \in \mathbb{F}_{q}\right\}$ for $t \in \mathbb{F}_{q}$. We have:

(i) if $m / d$ is even, then $\left|I_{f}(0)\right|=1+\frac{q-1}{2^{d}+1}$, and $\left|I_{f}(t)\right|=\frac{q+1}{2}+\frac{q-1}{2\left(2^{d}+1\right)}$ for all $t \in \mathbb{F}_{q}^{*}$; 
(ii) if $m / d$ is odd, then $\left|I_{f}(0)\right|=q$, and $\left|I_{f}(t)\right|=\frac{q-1}{2}+\frac{q+1}{2\left(2^{d}+1\right)}$ for all $t \in \mathbb{F}_{q}^{*}$.

Proof. For $t=0$, we have

$$
\left|I_{f}(0)\right|=1+\frac{2^{m}-1}{\operatorname{gcd}\left(2^{m}-1,2^{i}+1\right)}
$$

From the well-known fact (e.g. [8, Lemma 11.1]) that

$$
\operatorname{gcd}\left(2^{m}-1,2^{i}+1\right)= \begin{cases}1 & \text { if } m / d \text { is odd } \\ 2^{d}+1 & \text { if } m / d \text { is even }\end{cases}
$$

the assertion on $\left|I_{f}(0)\right|$ follows.

For $t \in \mathbb{F}_{q}^{*}$, by definition, we have

$$
\begin{aligned}
\left|I_{f}(t)\right| & =\left|\left\{f(x)+t x: x \in \mathbb{F}_{q}\right\}\right| \\
& =\left|\mathbb{F}_{q}\right|-\mid\left\{c \in \mathbb{F}_{q}^{*}: f(x)+t x+c \text { has no root in } \mathbb{F}_{q}\right\} \mid \\
& =q-N_{0}^{\prime} .
\end{aligned}
$$

To make use of Theorem 2, we transform $f(x)+t x+c$ following the steps in [1]. Since $t \neq 0$ and $c \neq 0$, let $x=\frac{c}{t} z$, then

$$
\begin{aligned}
& f(x)+t x+c \\
& =x^{2^{i}+1}+t x+c \\
& =\frac{c^{2^{i}+1}}{t^{2^{i}+1}}\left(z^{2^{i}+1}+\frac{t^{2^{i}+1}}{c^{2^{i}}} z+\frac{t^{2^{i}+1}}{c^{2^{i}}}\right) .
\end{aligned}
$$

Since

$$
\left\{\frac{t^{2^{i}+1}}{c^{2^{i}}}: c \in \mathbb{F}_{q}^{*}\right\}=\mathbb{F}_{q}^{*},
$$

we have $N_{0}^{\prime}=N_{0}$, where $N_{0}$ denotes the number of $b \in \mathbb{F}_{q}^{*}$ such that $x^{2^{i}+1}+b x+b$ has no root in $\mathbb{F}_{q}$. The conclusion then follows from Theorem 2 .

Proposition 3 shows that the smallest Kakeya sets constructed using Gold power functions are achieved with $i=m / 2$ for an even $m$, and $i=0$ for an odd $m$. The discussion below shows that the choice $i=m / 2$ implies a better upper bound on Kakeya sets compared with the one given in Theorem 1 . The idea to use $f(x)=x^{2^{m / 2}+1}$ to improve the bound in Theorem 1 appears in [6], and was independently suggested by David Speyer in [7]. Observe that $f(x)=x^{3}$ chosen in [5] to prove the bound for $m$ even is the Gold power function with $i=1$ and $d=1$.

When $m / d$ is odd, $\left|I_{f}(0)\right|=q$, and therefore the bound obtained by the Gold power functions cannot be good for large $n$. However, for small values of $n$, it is better than the one of Theorem 1 [6]. 
Next consider the function $f(x)=x^{2^{m / 2}+1}$. In particular, we show that this function yields a better upper bound on the minimum size of Kakeya sets in $\mathbb{F}_{q}^{n}$ when $q$ is an even power of 2. First we present a direct proof for the size of the sets $\left\{x^{2^{m / 2}+1}+t x: x \in\right.$ $\left.\mathbb{F}_{q}\right\}, t \in \mathbb{F}_{q}$.

Theorem 4. Let $m$ be an even integer. Then

$$
|I(0)|:=\left|\left\{x^{2^{m / 2}+1}: x \in \mathbb{F}_{q}\right\}\right|=2^{m / 2},
$$

and

$$
|I(t)|:=\left|\left\{x^{2^{m / 2}+1}+t x: x \in \mathbb{F}_{q}\right\}\right|=\frac{2^{m}+2^{m / 2}}{2}
$$

for all $t \in \mathbb{F}_{q}^{*}$.

Proof. The identity on $I(0)$ is clear, since the image set of the function $x \mapsto x^{2^{m / 2}+1}$ is $\mathbb{F}_{2^{m / 2}}$. Let $t \in \mathbb{F}_{q}^{*}$. Note that $|I(t)|=|I(1)|$. Indeed, there is $s \in \mathbb{F}_{q}$, such that $t=s^{2^{m / 2}}$ and then

$$
x^{2^{m / 2}+1}+t x=s^{2^{m / 2}+1} \cdot\left((x / s)^{2^{m / 2}+1}+(x / s)\right) .
$$

Hence it is enough to compute $I(1)$. Let $\operatorname{Tr}(x)=x^{2^{m / 2}}+x$ be the trace map from $\mathbb{F}_{q}$ onto its subfield $\mathbb{F}_{2^{m / 2}}$. Recall that $\operatorname{Tr}$ is a $\mathbb{F}_{2^{m / 2}}$-linear surjective map.

Set $g(x)=x^{2^{m / 2}+1}+x$. If $y, z \in \mathbb{F}_{q}$ are such that

$$
g(z)=z^{2^{m / 2}+1}+z=y^{2^{m / 2}+1}+y=g(y),
$$

then $z=y+u$ for some $u \in \mathbb{F}_{2^{m / 2}}$, since the image set of the function $x \mapsto x^{2^{m / 2}+1}$ is $\mathbb{F}_{2^{m / 2}}$. Further, for any $u \in \mathbb{F}_{2^{m / 2}}$

$$
g(y+u)=(y+u)^{2^{m / 2}+1}+y+u=y^{2^{m / 2}+1}+y+u\left(y^{2^{m / 2}}+y\right)+u^{2}+u .
$$

Hence, $g(y)=g(y+u)$ if and only if

$$
u\left(y^{2^{m / 2}}+y\right)+u^{2}+u=u(\operatorname{Tr}(y)+u+1)=0 .
$$

Consequently, two distinct elements $y$ and $z$ share the same image under the function $g$ if and only $\operatorname{Tr}(y) \neq 1$ and $z=y+\operatorname{Tr}(y)+1$. This shows that $g$ is injective on the set $\mathcal{O}$ of elements from $\mathbb{F}_{q}$ having trace 1, and 2-to-1 on $\mathbb{F}_{q} \backslash \mathcal{O}$, completing the proof.

Theorem 5. Let $q=2^{m}$ with $m$ even and $n \geqslant 1$. There is a Kakeya set $K \subset \mathbb{F}_{q}^{n}$ such that

$$
|K|<\frac{2 q}{q+\sqrt{q}-2}\left(\frac{q+\sqrt{q}}{2}\right)^{n} .
$$

Proof. The statement follows from (2) and Theorem 4. 


\section{On Kakeya sets constructed using the function $x \mapsto$ $x^{4}+x^{3}$}

In this section we obtain an upper bound on the minimum size of Kakeya sets constructed using the function $x \mapsto x^{4}+x^{3}$ on $\mathbb{F}_{q}$. For every $t \in \mathbb{F}_{q}$, let $g_{t}: \mathbb{F}_{q} \rightarrow \mathbb{F}_{q}$ be defined by

$$
g_{t}(x):=x^{4}+x^{3}+t x .
$$

Next we study the image sets of functions $g_{t}(x)$. Given $y \in \mathbb{F}_{q}$, let $g_{t}^{-1}(y)$ be the set of preimages of $y$, that is

$$
g_{t}^{-1}(y):=\left\{x \in \mathbb{F}_{q} \mid g_{t}(x)=y\right\} .
$$

Further, for any integer $k \geqslant 0$ put $\omega_{t}(k)$ to denote the number of elements in $\mathbb{F}_{q}$ having exactly $k$ preimages under $g_{t}(x)$, that is

$$
\omega_{t}(k):=\left|\left\{y \in \mathbb{F}_{q}:\left|g_{t}^{-1}(y)\right|=k\right\}\right| .
$$

Note that $\omega_{t}(k)=0$ for all $k \geqslant 5$, since the degree of $g_{t}(x)$ is 4 . The next lemma establishes the value of $\omega_{t}(1)$ :

Lemma 6. Let $q=2^{m}$ and $t \in \mathbb{F}_{q}^{*}$. Then

- if $m$ is odd

$$
\omega_{t}(1)= \begin{cases}\frac{q+1}{3} & \text { if } \operatorname{Tr}(t)=0 \\ \frac{q+4}{3} & \text { if } \operatorname{Tr}(t)=1\end{cases}
$$

- if $m$ is even

$$
\omega_{t}(1)= \begin{cases}\frac{q-1}{3} & \text { if } \operatorname{Tr}(t)=0 \\ \frac{q+2}{3} & \text { if } \operatorname{Tr}(t)=1\end{cases}
$$

Proof. Let $y \in \mathbb{F}_{q}$ and $y \neq t^{2}$. Then $t^{2^{m-1}}$ is not a solution of the following equation

$$
h_{t, y}(x):=g_{t}(x)+y=x^{4}+x^{3}+t x+y=0 .
$$

Observe that the number of the solutions for the above equation is equal to the one of

$$
\left(t^{2}+y\right) x^{4}+t^{2^{m-1}} x^{2}+x+1=x^{4} \cdot h_{t, y}\left(\frac{1}{x}+t^{2^{m-1}}\right)=0 .
$$

Hence either $\omega_{t}(1)$ or $\omega_{t}(1)-1$ is equal to the number of elements $y \in \mathbb{F}_{q}$ such that the affine polynomial

$$
\left(t^{2}+y\right) x^{4}+t^{2^{m-1}} x^{2}+x+1
$$

has exactly one zero in $\mathbb{F}_{q}$, depending on the number of preimages of $g_{t}(x)$ for $t^{2}$. Equation (4) has exactly 1 solution if and only if the linearized polynomial

$$
\left(t^{2}+y\right) x^{4}+t^{2^{m-1}} x^{2}+x
$$


has no non-trivial zeros, or equivalently

$$
u(x):=\left(t^{2}+y\right) x^{3}+t^{2^{m-1}} x+1
$$

has no zeroes.

Since $t^{2}+y \neq 0$, the number of zeroes of $u(x)$ is equal to the one of

$$
\frac{1}{t^{2}+y} \cdot u\left(\frac{1}{t^{2^{m-1}}} z\right)=\frac{1}{t^{2^{m-1}+1}}\left(z^{3}+\frac{t^{2^{m-1}+1}}{t^{2}+y} z+\frac{t^{2^{m-1}+1}}{t^{2}+y}\right) .
$$

Note that

$$
\left\{\frac{t^{2^{m-1}+1}}{t^{2}+y}: y \in \mathbb{F}_{q}, y \neq t^{2}\right\}=\mathbb{F}_{q}^{*} .
$$

Hence by Theorem 2 with $i=1$, the number of elements $y \in \mathbb{F}_{q}, y \neq t^{2}$, such that (5) has no zeros is

$$
\left\{\begin{array}{cl}
\frac{q+1}{3} & \text { if } m \text { is odd } \\
\frac{q-1}{3} & \text { if } m \text { is even. }
\end{array}\right.
$$

To complete the proof, it remains to consider $y=t^{2}$. In this case

$$
g_{t}(x)+y=x^{4}+x^{3}+t x+t^{2}=\left(x^{2}+t\right)\left(x^{2}+x+t\right),
$$

and therefore $g_{t}(x)+t^{2}$ has exactly one solution if $\operatorname{Tr}(t)=1$ and exactly 3 solutions if $\operatorname{Tr}(t)=0$.

Lemma 7. Let $q=2^{m}$ and $t \in \mathbb{F}_{q}^{*}$. Then

$$
\omega_{t}(3)= \begin{cases}1 & \text { if } \operatorname{Tr}(t)=0 \\ 0 & \text { if } \operatorname{Tr}(t)=1\end{cases}
$$

Proof. The proof of Lemma 6 shows that for any $y \neq t^{2}$, the number of solutions for $h_{t, y}(x)=0$ is a power of 2 . Hence only $t^{2}$ may have 3 preimages under $g_{t}(x)$, which is the case if and only if $\operatorname{Tr}(t)=0$.

The next lemma describes the behavior of the function $x^{4}+x^{3}$ :

Lemma 8. Let $q=2^{m}$ and $k \geqslant 1$ an integer. Then

- if $m$ is odd

$$
\omega_{0}(k)= \begin{cases}q / 2 & \text { if } k=2 \\ 0 & \text { otherwise, }\end{cases}
$$

in particular, the cardinality of $I(0):=\left\{x^{4}+x^{3}: x \in \mathbb{F}_{q}\right\}$ is $q / 2$. 
- if $m$ is even

$$
\omega_{0}(k)= \begin{cases}1 & \text { if } k=2 \\ \frac{2(q-1)}{3} & \text { if } k=1 \\ \frac{(q-4)}{12} & \text { if } k=4 \\ 0 & \text { otherwise. }\end{cases}
$$

Proof. Note that $x^{4}+x^{3}=0$ has 2 solutions. Let $y \in \mathbb{F}_{q}^{*}$. Then the steps of the proof for Lemma 6 show that the number of solutions of

$$
x^{4}+x^{3}+y=0
$$

is equal to the one of the affine polynomial

$$
a_{y}(x):=y x^{4}+x+1 .
$$

If the set of zeros of $a_{y}(x)$ is not empty, then the number of zeros of $a_{y}(x)$ is equal to the one of the linearized polynomial

$$
l_{y}(x):=y x^{4}+x .
$$

If $m$ is odd, then $l_{y}(x)$ has exactly 2 zeroes for every $y \neq 0$, implying the statement for $m$ odd. If $m$ is even, then $l_{y}(x)$ has only the trivial zero if $y$ is a non-cube in $\mathbb{F}_{q}$, and otherwise it has 4 zeroes. To complete the proof, it remains to recall that the number of non-cubes in $\mathbb{F}_{q}$ is $2(q-1) / 3$.

Lemmas 6-8 yield the following upper bound for the size of the image sets of the considered functions:

Theorem 9. Let $q=2^{m}$ with $m$ odd. For $t \in \mathbb{F}_{q}$ set $I(t):=\left\{x^{4}+x^{3}+t x: x \in \mathbb{F}_{q}\right\}$. Let $v$ be the number of pairs $x, z \in \mathbb{F}_{q}$ with $x^{2}+z x=z^{3}+z^{2}+t$. Then for $t \neq 0$

$$
|I(t)|=\frac{5}{8} q+\frac{q+1-v}{8}+\frac{\delta}{2}<\frac{5}{8} q+\frac{2 \sqrt{q}+5}{8},
$$

where $\delta=0$ or 1 if $\operatorname{Tr}(t)=0$ or 1 , respectively.

Proof. Note that

$$
|I(t)|=\omega_{t}(1)+\omega_{t}(2)+\omega_{t}(3)+\omega_{t}(4)
$$

and

$$
q=\omega_{t}(1)+2 \cdot \omega_{t}(2)+3 \cdot \omega_{t}(3)+4 \cdot \omega_{t}(4) .
$$

Let $v^{\prime}$ be the number of distinct elements $x, y \in \mathbb{F}_{q}$ with $x^{4}+x^{3}+t x=y^{4}+y^{3}+t y$. Clearly

$$
v^{\prime}=2 \omega_{t}(2)+6 \omega_{t}(3)+12 \omega_{t}(4),
$$


hence

$$
|I(t)|=\frac{5 q-v^{\prime}+3 \omega_{t}(1)-\omega_{t}(3)}{8} .
$$

Setting $y=x+z$, we see that $x^{4}+x^{3}+t x=y^{4}+y^{3}+t y$ for $x \neq y$ is equivalent to $x^{2}+z x=z^{3}+z^{2}+t$ for $z \neq 0$. However, for $z=0$ this latter equation has a unique solution, so $v=v^{\prime}+1$.

Together with Lemmas 6-8 we see that the size of $I(t)$ is as claimed. The inequality follows from the Hasse bound for points on elliptic curves, which in our case says that $|v-q| \leqslant 2 \sqrt{q}$. (Note that the projective completion of the curve $X^{2}+Z X=X^{3}+X^{2}+t$ has a unique point at infinity.)

The bound obtained in Theorem 9 can be stated also as follows

$$
|I(t)| \leqslant\left\lfloor\frac{5}{8} q+\frac{2 \sqrt{q}+5}{8}\right\rfloor,
$$

since $|I(t)|$ is an integer. Our numerical calculations show that for odd $1 \leqslant m \leqslant 13$ bound (6) is sharp, that is for these $m$ there are elements $t \in \mathbb{F}_{2^{m}}$ for which equality holds in (6).

Theorem 10. Let $q=2^{m}$ with $m$ odd and $n \geqslant 1$. There is a Kakeya set $K \subset \mathbb{F}_{q}^{n}$ such that

$$
|K|<\frac{8 q}{5 q+2 \sqrt{q}-3}\left(\frac{5 q+2 \sqrt{q}+5}{8}\right)^{n} .
$$

Proof. The statement follows from (2) and Theorem 9.

\section{References}

[1] A. W. Bluher. On $x^{q+1}+a x+b$. Finite Fields Appl., 10(3):285-305, 2004.

[2] Z. Dvir. On the size of Kakeya sets in finite fields. J. Amer. Math. Soc., 22(4):10931097, 2009.

[3] Z. Dvir. Incidence theorems and their applications. arXiv preprint arXiv:1208.5073, 2012.

[4] Z. Dvir, S. Kopparty, S. Saraf, and M. Sudan. Extensions to the method of multiplicities, with applications to Kakeya sets and mergers. In 2009 50th Annual IEEE Symposium on Foundations of Computer Science (FOCS 2009), pages 181-190. IEEE Computer Soc., Los Alamitos, CA, 2009.

[5] S. Kopparty, V. F. Lev, S. Saraf, and M. Sudan. Kakeya-type sets in finite vector spaces. J. Algebraic Combin., 34(3):337-355, 2011.

[6] G. Kyureghyan and Q. Wang. An upper bound on the size of kakeya sets in finite vector spaces. to appear in proceedings of WCC 2013.

[7] V. F. Lev. A mixing property for finite fields of characteristic 2, 2012. http://mathoverflow.net/questions/102751/ a-mixing-property-for-finite-fields-of-characteristic-2. 
[8] R. J. McEliece. Finite fields for computer scientists and engineers. The Kluwer International Series in Engineering and Computer Science, 23. Kluwer Academic Publishers, Boston, MA, 1987.

[9] G. Mockenhaupt and T. Tao. Restriction and Kakeya phenomena for finite fields. Duke Math. J., 121(1):35-74, 2004.

[10] S. Saraf and M. Sudan. An improved lower bound on the size of Kakeya sets over finite fields. Anal. PDE, 1(3):375-379, 2008.

[11] T. Wolff. Recent work connected with the Kakeya problem. In Prospects in mathematics (Princeton, NJ, 1996), pages 129-162. Amer. Math. Soc., Providence, RI, 1999. 